\title{
Reporting Mistreatment of Older Adults: The Role of Physicians
}

\author{
Dorrie E. Rosenblatt, MD, PhD, Kyung-Hwan Cho, MD, PhD, ${ }^{\dagger}$ and Paul W. Durance, PhD
}

OBJECTIVE: To characterize elder mistreatment reporting patterns over time and by reporting source with specific focus on physician reporting. To determine whether demographic or socioeconomic factors influenced the reporting of elder abuse in Michigan between 1989 and 1993 and whether these factors affected physician reporting rates.

DESIGN: Analysis of the State of Michigan's records of reported cases of suspected adult abuse for the years $1989-$ 1993.

MEASUREMENTS: Counties were categorized by size, urbanization, and average income. The study population was analyzed as four age groups: $18-64,65-74,75-84$, and 85-99. Physician to population ratios were calculated for the county types and compared with physician reporting rates.

MAIN RESULTS: A total of 27,371 cases of possible abuse were reported, 17,238 in persons older than age 65 . Physicians reported only $2 \%$ of cases, and physician reporting rates did not increase over the 5 -year period. Physician reporting rates were highest in small counties with low physician to population ratios. There was a high percentage of primary care physicians in these counties. Forty-seven percent of all reported cases were substantiated. There was no difference in substantiation rate for physician-reported cases compared with other professional reporting sources.

CONCLUSION: Physician reports average only $2 \%$ of all reports of suspected elder mistreatment. Primary care physicians in counties with low physician to population ratios appear to be more active in reporting mistreatment of older people. Increasing physician awareness of the problem of elder mistreatment and providing physicians with the tools to screen for mistreatment should increase the number of cases that are reported to the agencies responsible for assisting mistreated older people. J Am Geriatr Soc 44:65-70, 1996.

From the "Division of Geriatric Medicine, Department of Internal Medicine, and Institute of Cierontology, University of Michigan, and Geriatric Research Education and Clinical Center at the Ann Arbor VA Medical Center; ${ }^{\dagger}$ Department of Family Medicine, Korea University, Medical College, Postdoctoral Research Fellow, Division of Geriatric Medicine, Department of Internal Medicine, University of Michigan; and $\ddagger$ Research Health Scicnce Specialist, Ann Arbor Health Services Research and Development Field Program, Department of Veterans Affairs, Assistant Professor, Department of Health Management and Policy, School of Public Health, and consultant in the Methodology, Data Management and Analysis Core of the Older Americans Intervention Center, Institute of Gerontology, University of Michigan

Supported by Older Americans Intervention Center \#2 P60 A G08808-06. Address correspondence to Dorrie E. Rosenblatt, MD, Assistant Professor, University of Michigan Medical Center, Department of Internal Medicine, Division of Geriatric Medicine, 300 North lngalls, Ann Arbor, MI 48109-2007.
$\mathrm{E}_{\mathrm{s}}$ lder mistreatment is a catchall term that includes the harm types of abuse, neglect, exploitation, and endangerment. Congressional reports suggest that elder mistreatment is a problem that affects more than $4 \%$ of the older adults or close to two million older people in the United States.' All the states now have laws on elder mistreatment. ${ }^{2}$ Most states mandate reporting, and physicians are among those required to report suspected mistreatment. Yet the same congressional reports also estimate that only one in six cases is reported to the agencies charged with dealing with the problem. ${ }^{1}$ Other sources estimate that only one in 15 cases is reported. ${ }^{3} \mathrm{We}$ analyzed data from the Michigan Department of Social Services (DSS) for the years 1989-1993, with a focus on physician reporting, to see whether it was possible to identify regional, socioeconomic, or profession-specific obstacles to reporting of elder abuse.

The AMA has said that physicians, who are often the only people outside the family circle to have access to a frail older person, "are in an ideal position to recognize, manage, and prevent elder mistreatment." 4 Virtually all the states have laws mandating that physicians report elder mistreatment, and many states have penalties for failure to report. However, surveys by O'Brien, ${ }^{5}$ and Daniels, ${ }^{6,7}$ suggest that physicians are not knowledgeable about elder abuse legislation. Blakeley and Donlon ${ }^{8}$ asked workers in 241 Area Agencies on Aging to rate the helpfulness of 14 occupations in discovering abuse and neglect of older people. Physicians were rated as "not very helpful." Tatara" reviewed aggregate data on reporting from 29 states and found that $17 \%$ of cases were reported by physicians/health care professionals. The limited data that are available that specifically quantitate physician reporting indicate that only a small number of reports, in the range of 0.6 to $2 \%$ come from physicians. ${ }^{10,11}$

There are many obstacles to the recognition and management of elder mistreatment. A major obstacle has been the variety of definitions of the problem. ${ }^{12-14}$ Not only do the definitions of the harm types constituting elder mistreatment vary from state to state, they vary between the medical, legal, social work, and other professions involved in dealing with the problem. These conceptual problems in defining harm types and the lack of uniform definitions have hampered clinical, educational, and research efforts.

Another obstacle is denial of the problem. Whereas child abuse received attention in the 1960 s and spouse abuse received attention in the $1970 \mathrm{~s}$, it was not until the first reports of "granny-bashing" in the early 1980 s that elder mistreatment began to receive attention. ${ }^{15}$ A 1981 congressional report called elder abuse a "shameful and hidden 
problem". ${ }^{1}$ People do not like to think of older people being mistreated and so may fail to acknowledge a problem. The older people themselves are thought to refrain from complaining because of embarrassment, fear of retaliation, fear of nursing home placement or to protect the abuser, who is most commonly a child or spouse. ${ }^{16,17}$

Other barriers to detection and reporting of elder mistreatment are ageism and lack of knowledge. ${ }^{18-19}$ Many people, professionals included, expect older people to have a variety of problems - such as confusion, depression, falls, incontinence, etc. - and find it difficult to sort out what are the effects of normal aging, what is caused by disease, and what is attributable to neglect or abuse. ${ }^{14}$ Lack of knowledge about elder mistreatment, about the laws relating to it, and about the resources available for providing assistance to victims, also play a role in reducing reporting. ${ }^{20}$

All of these factors impact on physician reporting of elder mistreatment. Other explanations offered for the low physician reporting rate include a failure to recognize the problem, hesitancy to report for fear of making the situation worse, a wish to maintain a relationship with the patient and family, avoidance of potential court involvement, and a lack of belief that state intervention will result in a better outcome. $^{21}$

\section{METHODS}

Michigan defines harm types as abuse (nonaccidental physical, mental, or sexual maltreatment), neglect (failure to provide adequate food, clothing, shelter, or medical care), exploitation (misuse of funds, property or dignity) and endangerment (a life threatening situation caused by the inability of the threatened person to respond).

The state of Michigan collects data from all counties annually on the reporting of elder mistreatment. Between 1989 and 1993, there were 27,371 reports of possible mistreatment in adults aged 18 to 99 . We analyzed these data to determine reporting patterns, with a focus on physician reporting of elder mistreatment. In an effort to identify variables that could serve as the basis for interventions to increase reporting rates, we assessed the impact on reporting rates of county socioeconomic characteristics, harm type, age group, and physician to population ratio,

The 1990 Census data provided the county demographic data for the study. To determine the impact of socioeconomic factors on elder mistreatment reporting rates we categorized the 83 counties in Michigan by size, urbanization, and average income. The counties were assigned to four groups based on population and urbanization. By this definition, the population cut-offs for each county type were metropolitan: 200,000; medium urban and medium rural: 30-200,000; small less than 30,000 . Medium size counties were defined as urban if more than $50 \%$ of the population was classified as urban on the US census. Counties were further subdivided by average income into rich $(<10 \%$ below poverty line), medium, and poor ( $>19 \%$ below poverty line), again based on census data.

For study purposes the DSS data was analyzed for four age groups: 18 to 64,65 to 74,75 to 84 , and 85 to 99 because birth century was not available and we could not determine reliably ages 100 and older. The 1990 census provided the data on the distribution of in each age group in the counties, and the 1993 American Medical Association listing of non- federal physicians by state and county provided data on physician numbers.

Stepwise multivariate linear regression was used to identify factors impacting on reporting rates. In interpreting the results, we take into account the potential for small effects to acquire significance in a large data set such as this one.

The 27,371 reported cases of possible mistreatment in adults aged 18 to 99 between 1989 and 1993 from the state of Michigan were analyzed.

\section{RESULTS}

By our definitions there are nine metropolitan, 10 medium urban, 28 medium rural, and 36 small counties. The older population (over age 65) was distributed fairly evenly over the county types although there was a trend toward a higher percentage of the population falling into older age groups in the small counties. When the counties were classified by wealth the distribution of older people was also quite even. This even distribution allowed comparison of physician reporting patterns across county types.

During the 5-year period studied, there were 28,025 reports to the Department of Social Services. A complete data set was available for 27,371 cases, of which 17,238 were in persons older than 65 years of age. Thirty-seven per cent of the reports were made on persons less than age 65 . The three older groups- 65 to 74,75 to 84 , and 85 to 99 -made up $18.6 \%, 26.3 \%$, and $18.2 \%$ of the total, respectively. Agespecific reporting rates clearly increase with increasing age from $0.4 / 1000$ in the age 18 to 64 age group to $11.0 / 1000$ in the 85 to 99 -year-old group.

Reporting became mandatory in Michigan in 1985. The total number of reports fluctuated during the 5 -year period between 1989 and 1993 although there was a small upward trend.

Analysis of reporters showed that physicians reported only about $2 \%$ of the cases. Twenty-six per cent of referrals came from non-physician health care providers, $41 \%$ from the community, $25 \%$ from social work and mental health, and $5.0 \%$ from law enforcement. Comparison of reporting rates over the 5 -year period (Figure 1) showed that the physician reporting rate did not improve with time, although reporting by other health care workers increased.

We assessed the impact of physician to population ratio to determine whether physician scarcity contributed to low physician reporting rate. Figure $2 \mathrm{~A}$ shows the relationship of physician to population for each county type and its impact on physician reporting. There was actually an inverse relationship between number of physicians per 1000 population served and the number of physician reported mistreatment cases. Analysis of physician specialty, Figure 2B, showed that primary care physicians constituted a high percent of the physicians in the counties with low physician to population ratios and higher physician reporting rates. The older age distribution in small counties was not a contributor since chi-square analysis of physician reporting rates in persons 18 to 64 and 65 and older showed no differences $(P=.142)$ between the younger and older age groups.

Comparison of substantiation rates showed no significant differences between physician reports and other professional reporting sources. Overall, 12,673 cases were substantiated. The average substantiation rate was $47 \%$ (range $0-100)$. The data set provides a harm type only for substantiated cases. Analysis of harm types for physician-reported 


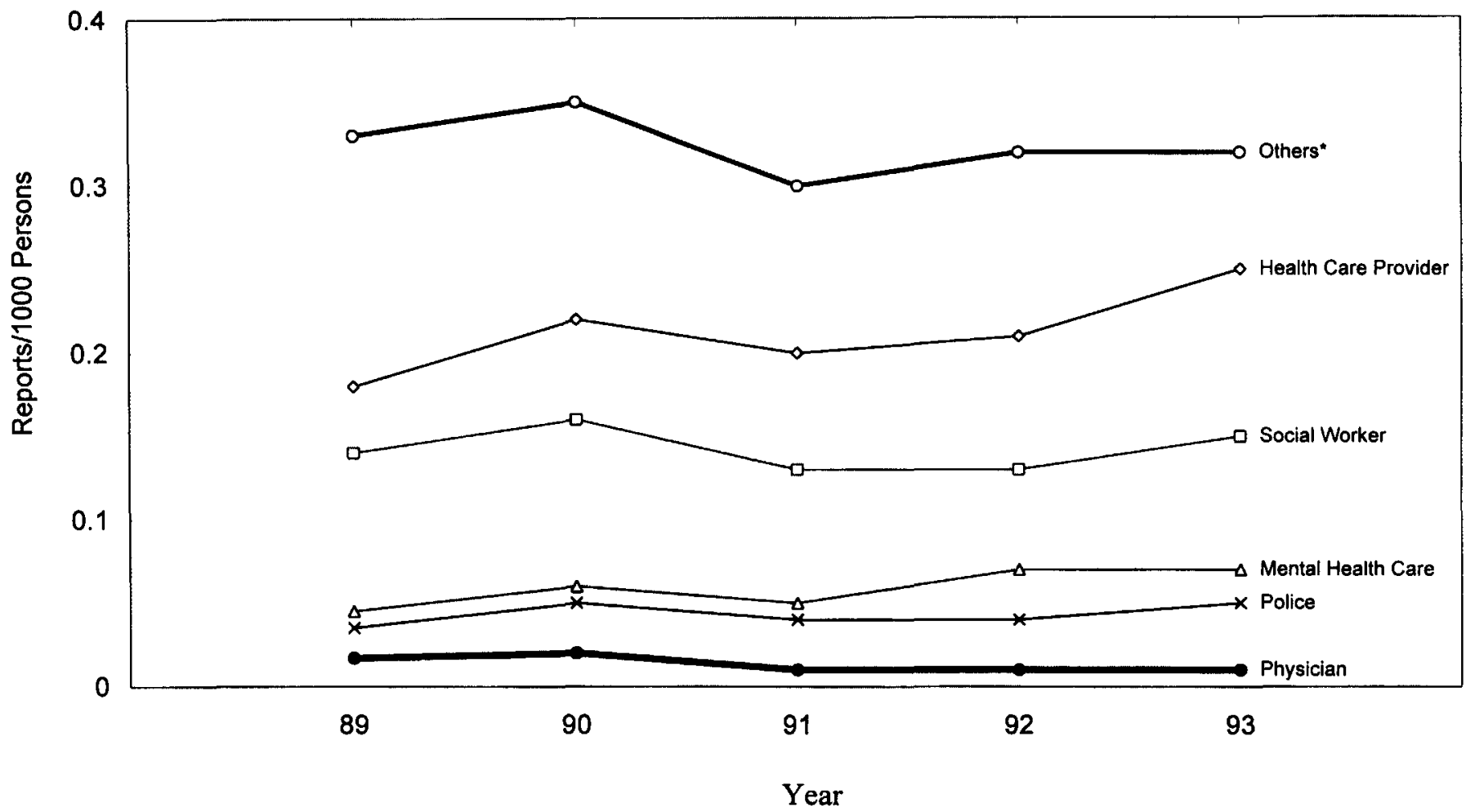

Figure 1. Reporting rates from different reporting sources between 1989 and 1993 . Physicians reported only $2 \%$ of the cases. The category "health care provider" includes nurses and home health aides. The category "other" includes relatives, friends, concerned citizens, and a few professionals (such as clergy) not included in the groups shown in the figure.

cases showed that physicians were more likely to report abuse and least likely to report exploitation.

\section{DISCUSSION}

Our data represent yearly reports to the DSS from all 83 counties in Michigan (population 9,295,797) for the years 1989-1993. We analyzed the 27,371 usable reports of abuse and the 12,673 substantiated case reports to determine whether there were identifiable regional or socioeconomic factors that influenced reporting of suspected elder mistreatment. We were particularly interested in the role of physicians in reporting elder mistreatment. To assess the effects of population size, urbanization, and average income, we divided the counties into 11 categories (there were no poor, medium urban counties). The older population was relatively evenly divided among county types although there was a small trend toward higher percentages of older people in the smaller counties. This probably reflects the lack of employment in these low population areas and the migration of younger people to areas with better employment potential. There was equal distribution of older adults across county wealth types. This equal distribution of older adults permitted us to analyze the impact of socioeconomic factors and physician to population ratio on the reporting of elder mistreatment.

Reporting of suspected elder mistreatment became mandatory in Michigan in 1985, so our data set covers a period starting 4 years after the legislation. The absolute number of reports fluctuated during the 5 -year period. We did not see a consistent increase in reporting rates although there were $19.4 \%$ more cases reported in 1993 than in 1989. This finding is of concern inasmuch as other states have shown a steady increase in reports over a similar time period. NARCEA has estimated a $94 \%$ increase in aggregate state reports between 1986 and 1991. ${ }^{25}$ (It should be noted that these increases are calculated based on total reports and not on the age-adjusted reporting rate as we have calculated it). In contrast, reporting of child abuse increased for years after the passing of legislation mandating reporting. The reporting rate for child abuse has been estimated at 27.3/1000, and the number of reports increased $158 \%$ between 1976 and $1984 .^{26}$ The National Center on Child Abuse Prevention cites a reporting rate of 45/1000 in 1993 and estimated a steady increase of $6 \%$ per year between 1988 and $1993 .{ }^{27}$ It is thought that one of three cases of child abuse is reported compared with 1 in 5 to 1 in 15 for elder abuse. ${ }^{1}$ There is no mandatory reporting for spouse abuse, but it has been estimated at about 2,000,000 cases per year or approximately $3.8 \%$ of all wives (or spouse equivalents). ${ }^{28}$ The marked difference in the likelihood that a needy older person will be reported compared with a child, and the lack of growth in reporting rates for elder mistreatment, should be a major concern in our aging society.

In Michigan, the majority of reports came from citizens $(41 \%)$, nursing $(26 \%)$, and social work $(18 \%)$; only $2 \%$ of reports came from physicians. This number is at the upper end of the range of reporting rates from other states. It is still unclear why physicians, who should be the gatekeepers for so many of the other reporting professions, have so low a reporting rate. Surveys have suggested that physicians are lacking in awareness of reporting law and procedures. ${ }^{6,7}$ But the AMA and state medical societies have been making efforts at raising physicians' awareness of the problem. One obstacle to physician recognition of elder mistreatment is that, al- 

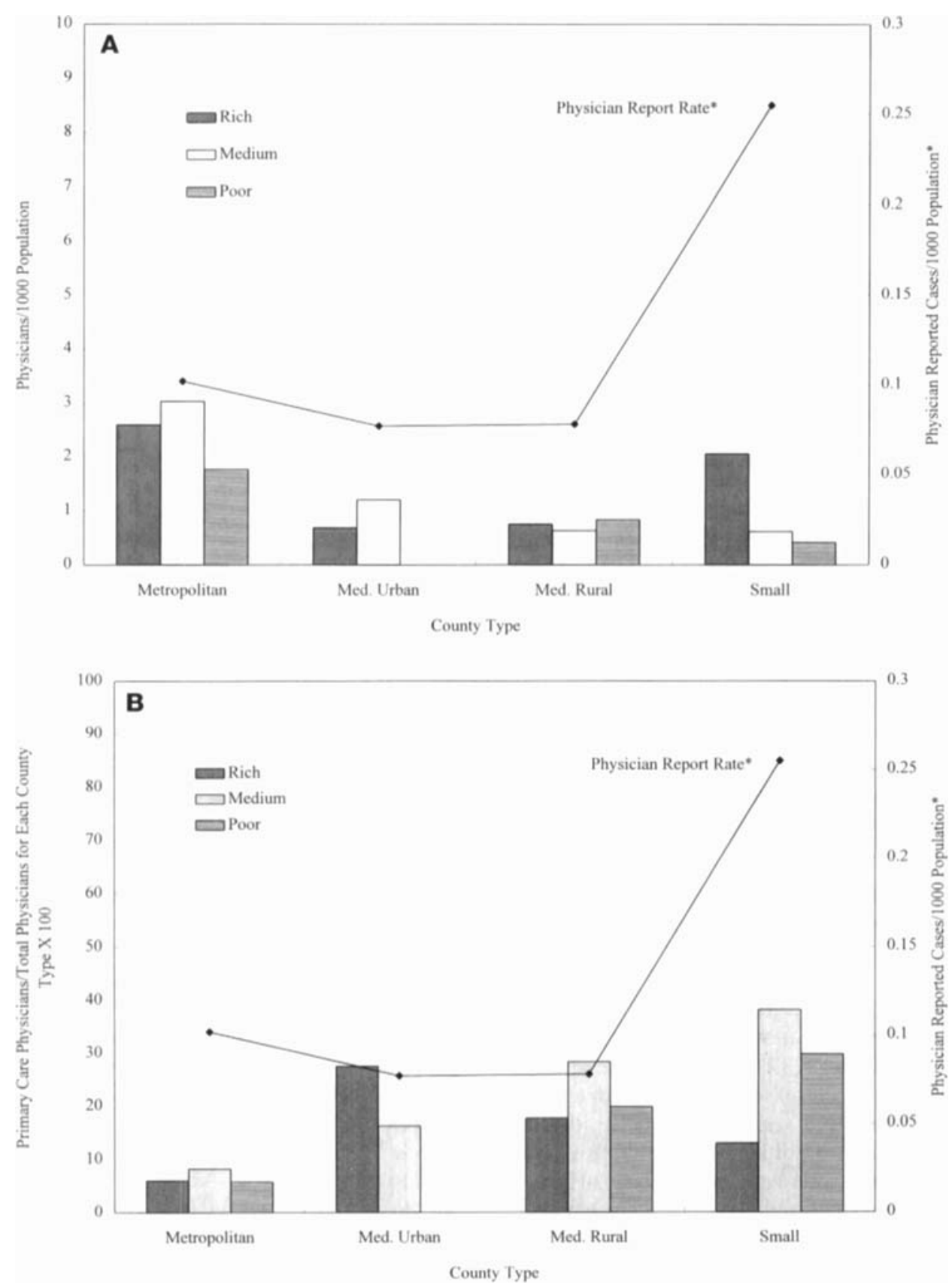

Figure 2. A, The relationship between the physician to population ratio for the four county size types and physician reporting rates for elder abuse in those county types. Physician reporting rates were highest in the small counties.

$\mathrm{B}$, Relationship between the physician reporting rate and the percentage of primary care physicians in each county type. Counties with a higher percentage of primary care physicians had a higher reporting rate.

though there are several protocols which have been published for emergency room screening for abuse, there is no brief, well validated screen for use in primary care. Johnson ${ }^{29}$ has reviewed the problems in developing screen tools. This lack of tools, especially in combination with the time limits on physician patient interactions imposed by the realities of Medicare reimbursement, may result in less than ideal physician recognition of the problem of elder mistreatment. It is also possible that physicians are recognizing elder abuse but either electing not to report it or delegating the reporting to others. Suggested reasons why physicians may elect not to report include fear of legal involvement, maintaining a rela- tionship with the patient and caregiver, unfamiliarity with reporting procedures, a perception that reporting will make things worse, and concern about the adequacy of state agency responses. Interestingly, a study of nurses and physicians in Alabama ${ }^{21}$ showed that nurses, who in most studies seem to report more elder mistreatment than physicians, were much more concerned than the physicians that state agency responses might not be adequate.

It was interesting that the physician reporting rate was inversely proportional to the physician to population ratio. An explanation for this may be that the physicians in underserved areas tend to be primary care (internists or family 
practice) physicians who may be more attuned to looking for elder abuse than specialists. They may also know their community and patient population better than physicians in larger areas. A similar pattern of higher physician reporting rates for child abuse has been demonstrated in rural areas.

We compared the types of cases likely to be reported by physicians with cases reported by other sources. Physicians were most likely to report physical abuse. This may reflect cases seen in the emergency room setting. Physicians were least likely to report exploitation. This is understandable as few practicing physicians have time to investigate in detail their patients psychosocial problems.

To determine whether, when they did report, physicians were reporting the most obvious cases, we compared substantiation rates for the different reporting sources. There was no difference in the substantiation rate for physicianreported cases. Intuitively, one might think that certain professions, such as visiting nursing, might have more expertise and a higher substantiation rate than, for example, concerned citizens. In fact, Sharon ${ }^{30}$ has shown higher substantiation rates for cases reported by professionals in Wisconsin. In Michigan there was a small trend toward higher substantiation of cases reported by professionals compared with those reported by "others" (odds ratio 0.84 ). However, the overall substantiation rate is lower than that in Wisconsin, and the differences in Michigan substantiation rates for professionals and "others" is much smaller than that seen in Wisconsin. The small differences in substantiation rates may indicate that all Michigan reporters have a high threshold for reporting and so all reporters wait until a case is equally obvious. If this is true it would seem that the substantiation rate should be considerably higher than $47 \%$. There is no clear explanation for the differences in substantiation rates between states.

Data from this study do not provide incidence numbers for elder mistreatment because it is unclear what percentage of cases were reported. There is very limited data on the prevalence of abuse in the community. The existing studies consist of small surveys of older adults asked about their own experiences ${ }^{31,32}$ or surveys of professionals or older people asking if they have seen any cases of elder abuse. These studies have been reviewed by McDonald et al. ${ }^{33}$ and by Pillemer and Wolf. ${ }^{34}$ The survey data suggest an incidence rate for elder mistreatment of 4/1000. For Michigan, this would calculate out to 27,334 cases, compared with the 6105 cases actually reported in 1993 . This equals a reporting rate of 1 in 5 possible cases, which is in the mid-range of reporting estimates from around the country.

Clearly, Michigan and all the other states have a way to go in detecting and reporting elder mistreatment. Part of the process needed to improve reporting rates is education. However, although the AMA and other organizations have made significant efforts to educate physicians on elder abuse, physician reporting rates are still quite low $(2 \%)$. This is of concern because physicians are often the only persons outside of the family to see at risk older adults and because physicians, to a large extent, control access of other professions to older people since a physician prescription is required before Medicare or Medicaid will reimburse services. More education about elder mistreatment, its recognition, management, and prevention, is clearly needed for physicians, other professional groups, and the public. Physicians should take a leading role both in educational programs and in initiating the research needed to provide the foundation for educational and legislative efforts.

Another very important challenge is the development of screening instruments for elder abuse that are easily usable in primary care practice. Nearly all of the currently published screens are based on emergency room findings, and this is a special subpopulation of abused older adults. Primary care physicians need better tools for detecting potential elder mistreatment. The AMA has suggested a list of screening questions, ${ }^{4}$ but these, although practical, have yet to be validated. Physicians should take a lead in designing and validating screening instruments and then in using the screens routinely in their work with frail older persons.

The AMA and other organizations have been trying to educate physicians about elder mistreatment and reporting procedures. Surveys suggest that if physician reporting rates are to improve, the states must make an effort to convince the medical profession that reporting of possible elder mistreatment results in improved outcomes for the patients. It is well known that in many states, the agencies responsible for responding to reports of elder mistreatment have limited staff and limited budgets. This precludes ideal responses to the needs of older victims of mistreatment. Physicians can help the states to respond better by advocating for funding for the agencies responsible for dealing with elder mistreatment. Physicians should also try to influence policy and funding for services for older people at the federal level.

\section{CONCLUSION}

Elder mistreatment is a common problem and affects some 2 million older people in this country. Our study shows that Michigan has had 17,238 reported cases in persons aged 65 and older during the 5-year period between 1989 and 1993. The data suggest that only one case in five may be brought to the attention of agencies responsible for adult protective services. Physicians potentially have the best opportunities of any profession to detect, manage, and prevent elder mistreatment because they are often the only contacts for frail older people other than the caregivers, who are often the perpetrators of elder mistreatment. Yet, our findings suggest that physicians are not living up to their potential, i.e., they report less than $2 \%$ of all cases. Our data suggest that rural primary care physicians do best at detecting and reporting elder mistreatment. All physicians need to educate themselves and others about elder mistreatment, reporting procedures, coordination of care management with responsible agencies, and prevention. The development and use of screening instruments would give busy primary care physicians a better opportunity to detect elder mistreatment. Physicians should be part of the process of developing those instruments so that they are clinically practical. Physicians should also join groups advocating for adequate funding for the agencies responsible for providing services for older people and for dealing with elder mistreatment. In her 1993 article, "Elder Abuse Ten Years Later", ${ }^{35}$ Wolf challenged physicians to play a larger role in all of these areas essential to addressing the problem of elder abuse. Our data suggest that we physicians need to make a greater effort to meet that challenge.

\section{ACKNOWLEDGMENTS}

The authors thank Mr. Bill Chaliman of the Department of Social Services for his assistance in providing the data from the State of Michigan. 


\section{REFERENCES}

1. Elder Abuse (An Examination of a Hidden Problem). A Report (With Additional Views). Select Committee on Aging, US House of Representatives, Ninety-Seventh Congress, First Session. April 8, 1981.

2. Brewer RA, Jones JS. Reporting elder abuse: Limitations of statutes. Ann Emerg Med 1989;18:11:127-131.

3. American Public Welfare Association. Testimony of Toshio Tatara on Behalf of NARCEA. Washington, DC: American Public Welfare Association, 1989. P 25.

4. Diagnostic and Treatment Guidelines on Elder Abuse and Neglect. Chicago: American Medical Association, 1992.

5. O'Brien JG. Elder abuse: Barriers to identification and intervention. Presented at the Annual Meeting of the Gerontological Society of America, Chicago, March 1986.

6. Daniels RS, Baumhover LA, Clark-Daniels CL. Physicians mandatory reporting of elder abuse. Gerontologist 1989;29:321-327.

7. Clark-Daniels C, Daniels RS, Baumhover LA. Abuse and neglect of the elderly: Are emergency department personnel aware of mandatory reporting lows? Ann Emerg Med, 1990;19:49-56.

8. Blakely BE, Donlon $R$. The relative contributions of occupation groups in the discovery and treatment of elder abuse and neglect. J Gerontol Soc Work 1991:17:183-199.

9. Tatara $T$. Understanding the nature and scope of domestic elder abuse with the use of state aggregate data: A summary of the key findings of a national survey of state APS and aging agencies. J Elder Abuse Neglect 1993;5:35-57.

10. Cochran C, Petrone S. Elder abuse: The physician's role in identification and prevention. IL.L. Med J 1987;171:241-246.

11. Cash T, Valentine D. A decade of adult protective services: Case characteristics. J Gerontol Soc Work 1987;10:47-60.

12. Valentine D, Cash T. A definitional discussion of elder maltreatment. J Gerontol Soc Work 1986;9:17-29.

13. Breckman LS, Adelman RD. Elder mistreatment defined. In: Breckman LS, Adelman R. eds. Strategies for Helping Victims of Elder Mistreatment. Sage Human services Guide 53. Newbury Park, CA: Sage, 1988, pp 11-22.

14. Fulmer TT, O'Malley TA. The difficulty of defining abuse and neglect. In: Fulmer TT, O'Malley TA, eds. Inadequate Care of the Elderly. New York: Springer Publishing Company, 1987, pp 13-24.

15. Bennett $G$, Kingston $P$. Historical background: Definitions and theories. In Bennett $G$, Kingston $P$, eds. Elder Abuse, Concepts, Theories and Interventions. London: Chapman and Hall, 1993, pp 1-22.

16. Costa AJ. Elder abuse. Prim Care 1993;20:375-389.
17. Tomita SK. The denial of elder mistreatment by victims and abusers: The application of neutralization theory. Violence Vict 1990;5:171-184.

18. Breckman RS, Adleman RD. Barriers to detection. In: Breckman RS, Adleman RD, eds. Strategies for Helping Victims of Elder Mistreatment. Sage Human Services Guide 53, Newbury Park, CA: Sage, 1988, pp 32-43.

19. Council Report. Elder abuse and neglect. JAMA 1987;257:966-971.

20. Blakely BE, Donlon R, May DD. Improving the responses of physicians to elder abuse and neglect: Contributions of a model program. J Gerontol Soc Work 1993;19:35-46.

21. Clark-Daniels CL, Daniels RS, Baumhover LA. Physicians' and nurses' responses to abuse of the elderly: A comparative study of two surveys in Alabama. J Elder Abuse Neglect 1989; 1:57-72.

22. Ohio physicians' elder abuse prevention project. Ohio State Medical Association/The Ohio Medical Education and Research Foundation, Columbus OH, 1994.

23. South Carolina Omnibus Adult Protective Services Act 1993.

24. Benton D, Marshall C. Elder abuse. Clin Geriatr Med 1991;7:831-845.

25. Tatara T. Summaries of the statistical data on elder abuse in domestic settings for FY 90 and FY 91. Washington, DC: NARCEA, 1993.

26. Hampton RL. Physical victimization across the life span: Recognition ethnicity, and deterrence. In: Straus MB, ed. Abuse and Victimization Across the Life Span, Baltimore: Johns Hopkins Univ. Press, pp 203-222.

27. McCurdy K, Daro D. Current trends in child abuse reporting and fatalities. National Center on Child Abuse Prevention Research, Publication No. 808 Chicago, IL, 1994.

28. American Medical Association diagnostic and treatment guidelines on domestic violence. Arch Fam Med 1992;1:39 -47.

29. Johnson TF. Elder mistreatment identification instruments: Finding common ground. In: Johnson TF, ed. Elder mistreatment: Deciding Who Is at Risk. Westport, CT: Greenwood Press, 1991, pp 85-115.

30. Sharon N. Elder abuse and neglect substantiations: What they tell us about the problem. J Elder Abuse Neglect 1991;3:19-35.

31. Pillemer K, Finkelhor D. The prevalence of elder abusc: A random sample survey. Gerontologist 1988:28:51-57.

32. Podnieks E. National survey on abuse of the elderly in Canada. J Elder Abuse Neglect 1992;4:5-58.

33. McDonald PL, Hornick JP, Robertson GB. The definition, context, and prevalence of elder abuse and neglect. In: McDonald PL, Hornick JP, Robertson GB, Wallace JE, eds. Elder Abuse and Neglect in Canada. Butterworths Canada Ltd., 1991, pp 1-20.

34. Pillemer $K$, Wolf $R$, eds. Elder abuse: Conflict in the family. Dover, MA: Auburn House Publishing Company, 1986, pp 129-148.

35. Wolf RS. Elder abuse: Ten years later. J Am Geriatr Soc 1988;36:758-762. 